\title{
Utilidad del rastreo de anormalidades urinarias mediante tirillas reactivas en adultos jóvenes
}

\author{
Screening for urinary tract disease using urine dipstick in young adults
}

Vivante A y col. JAMA 2011;306(7):729-736

\section{Objetivos}

Evaluar la utilidad del rastreo de anormalidades urinarias asintomáticas mediante tirillas reactivas en adultos jóvenes.

\section{Diseño, lugar y pacientes}

Estudio de cohortes que incluyó datos de 1,2 millones de jóvenes de 16 a 25 años de ambos sexos en Israel. Se monitoreó el desarrollo de nefropatía crónica con requerimiento de tratamiento de diálisis y/o transplante renal.

\section{Evaluación de factores pronósticos y medición de resulta- dos principales}

Se evaluó la prevalencia de anormalidades urinarias asintomáticas. Se calculó el hazard ratio* $(\mathrm{HR})$ de aparición de enfermedad renal crónica terminal (ERCT) asociado a la presencia de microhematuria.

\section{Resultados principales}

Se diagnosticó hematuria microscópica aislada, asintomática y persistente en 3.960 individuos de un total de $1.203 .626(0,3 \%$ global; $0,4 \%$ en hombres y $0,2 \%$ en mujeres). Durante un seguimiento de 21,88 años (DS 6,74), desarrollaron ERCT el $0,70 \%$ de dichos individuos, mientras que sólo lo hicieron el $0,045 \%$ de los individuos con sedimento urinario normal. El $\mathrm{HR}^{*}$ de los portadores de microhematuria para desarrollo de ECRT ajustado por edad, sexo, país de origen paterno, año de enrolamiento, índice de masa corporal y presión arterial basal fue de 18,5 (IC 95\%: 12,4 a 27,6).

\section{Conclusiones}

La presencia de hematuria microscópica asintomática aislada en personas de 16 a 25 años se asoció con un aumento significativo de ERCT durante un seguimiento de 22 años, sin embargo su incidencia y riesgo absoluto son muy bajos.

Fuente de financiamiento: no referida.

\section{Comentario}

Se considera microhematuria a la presencia de más de tres hematíes por campo en el examen microscópico del sedimento urinario, en por lo menos dos ocasiones alejadas de la menstruación, ejercicio, trauma o actividad sexual recientes. La presencia de hematíes dismórficos $(>20 \%)$, acantocitos (> $1 \%$ ), cilindros hemáticos $(\geq 1)$, proteinuria $(>0,20 \mathrm{~g} /$ día $)$ y caída de filtrado glomerular aumentan la sospecha de enfermedad renal y la eventual necesidad de realizar una biopsia renal. Por el contrario, la sospecha de una hematuria urológica (coágulos, hematíes eumórficos) orienta a la evaluación estructural renal y de la vía urinaria (imagen, endoscopía y/o citología) sobre todo en personas mayores de 40 años de edad. Los pacientes portadores de microhematuria asilada pueden desarrollar con el tiempo hipertensión arterial, proteinuria y nefropatía ${ }^{1}$.

El uso de tirillas reactivas también pueden utilizarse para la detección de proteinuria en orina. Es importante destacar el carácter semicuantitativo del método, dependiente del observador, que puede detectar concentraciones de proteinuria superiores a $30 \mathrm{mg} / \mathrm{dL}$ pero que no es útil para el rastreo de microalbuminuria, ni de cadenas livianas, las cuales si son detectadas cuando se realiza dosaje de albuminuria y proteinuria respectivamente. Cabe mencionar además que se ha demostrado una buena correlación entre la proteinuria de 24 hs y el cociente proteinuria/creatininuria en muestra aislada de orina matinal ${ }^{1}$.

Estudios distintos del aquí analizado han documentado una prevalencia de anormalidades urinarias asintomáticas del 0,18 a 16,1 \% en la población general, así como la asociación de la proteinuria con un riesgo aumentado no sólo de ERCT, sino además enfermedad cardiovascular y mortalidad general. Sin embargo entidades como la Fuerza de Tareas preventiva de los EE.UU. (Sigla en inglés, USPSTF) han catalogado al rastreo de enfermedad renal crónica (utilizando por ejemplo la estimación del filtrado glomerular y la determinación de microalbuminuria) como "l" (de tipo insuficiente para evaluar el balance entre los beneficios y daños potenciales del rastreo) $)^{2}$.

Si bien el presente estudio demuestra un riesgo relativo significativamente incrementado de desarrollar ERCT entre los pacientes con microhematuria asintomática aislada seguidos durante 22 años, el riesgo absoluto de desarrollar tal condición resulta sumamente bajo. Resulta además importante considerar el efecto perjudicial que podría tener el rastreo sobre los pacientes con resultados falso positivos (etiquetamiento, estudios complementarios adicionales, etc.).

\section{Conclusiones del comentador}

Al momento actual no existen elementos suficientes como para considerar el rastreo de ERCT mediante la detección de microhematuria detectada mediante tirillas reactivas en la población general de adultos jóvenes.

Carlos G. Musso [ Servicio de Nefrología del Hospital Italiano de Buenos Aires. carlos.musso @ hospitalitaliano.org.ar ]

Musso CG. Utilidad del rastreo de anormalidades urinarias mediante tirillas reactivas en adultos jóvenes. Evid Act Pract Ambul. Abr-Jun 2012:15(2): 51. Comentado de Vivante A, Afek A, Frenkel-Nir Y, et al. Persistent Asymptomatic Isolated Microscopic Hematuria in Israeli Adolescents and Young Adults and Risk for End-Stage Renal Disease. JAMA 2011;306(7):729-736. PMID 21846854.

\section{Referencias}

1. Lerma E, Nissenson A. Nephroloy secrets. Philadelphia. Elsevier. 2011

2. USPSTF. Screening for chronic kidney disease: U.S. Preventive Services Task Force. Recommendation Statement Draft (en línea). Disponible en URL: http://www.uspreventiveservicestaskforce.org/uspstf12/kidney/draftrecckd.htm (ultimo acceso 10/06/2012). 